\title{
Cryptosporidiosis in persons with HIV infection
}

\author{
Bharat Ramratnam, Timothy P Flanigan
}

\begin{abstract}
Summary
Cryptosporidium parvum is an ubiquitous protozoan parasite that is a major cause of diarrhoea in individuals infected with human immunodeficiency virus. The hallmarks of infection include profuse watery diarrhoea which may become chronic in the severely immunosuppressed individual. No uniformly effective therapy exists. Current treatment relies upon a trial of anti-retroviral and specific anti-cryptosporidial medications, adequate fluid and nutritional support, and antimotility agents.
\end{abstract}

Keywords: cryptosporidiosis, AIDS, HIV

Cryptosporidium: structure and taxonomy

- coccidian protozoan parasite assigned to class Sporozoa in the order Eucoccidiida

- taxonomically related to other coccidia including Toxoplasma gondii, Isospora belli and Plasmodium

Oocysts

- extremely small $(2-5 \mu \mathrm{m}$ in diameter)

- using acid-fast stains, appear red with variable intensity

- resistant to standard disinfectants and chlorine

Box 1

The Miriam Hospital, Brown

University School of Medicine,

Providence, RI, USA

B Ramratnam

TP Flanigan

Correspondence to Bharat Ramratnam, MD, 450 East 63rd Street, Apt 2F, New York, NY10021, USA

Accepted 29 January 1997
Cryptosporidium parvum is a coccidian protozoan parasite that belongs to the same family as Isospora and Toxoplasma. ${ }^{1}$ Since its discovery by Tyzer in 1907 and until the advent of autoimmune deficiency syndrome (AIDS), Cryptosporidium was primarily regarded as a cause of diarrhoea among animals. ${ }^{2}$ Indeed, before 1982, only seven cases of human cryptosporidiosis had been reported in the medical literature. The last 15 years has witnessed a dramatic increase in the number of cases, owing to better diagnostic methods and a growing population of individuals infected with human immunodeficiency virus (HIV). ${ }^{3}$

\section{Aetiology}

Symptoms of cryptosporidial infection begin 5-22 days after ingestion of as few as 30 oocysts of human, animal or environmental origin. ${ }^{4}$ After ingestion, oocysts excyst and release sporozoites, the infective form of the parasite. The sporozoites implant on the mucosal surface of host epithelial cells and undergo sexual and asexual reproduction with generation of oocysts and merozoites, respectively. Merozoites continue the process of mucosal auto-infection while oocysts are immediately infectious and are excreted in stools. Note that the entire life-cycle takes place within a single host. ${ }^{1,2}$ Though the most common site of infection is the gut, extra-intestinal infection has been reported rarely, most commonly in the respiratory tract. ${ }^{5}$

The precise mechanism by which Cryptosporidium causes diarrhoea is unknown. Ultrastructural studies reveal that, while the parasite attaches to the mucosal epithelial lining, it does not invade the submucosa. ${ }^{6}$

Mechanisms that promote clearance of $C$ parvum remain unclear. On the basis of clinical and experimental data, it is generally accepted that both the humoral and cellular immune systems are required for control of infection. ${ }^{7}$ Studies using several animal models suggest that loss of T-lymphocyte function may lead to persistent cryptosporidiosis. ${ }^{8}$ The importance of B-cell-mediated immunity is suggested by the fact that immunocompromised persons with hypo-gammaglobulinaemia or isolated IgA deficiency may develop chronic cryptosporidiosis. ${ }^{9,10}$

\section{Epidemiology}

Cryptosporidium is an ubiquitous parasite and infections have been traced to household or sick contacts, agricultural exposure, and contaminated water and food. The ease with which humans become infected is illustrated by attack rates of up to $70 \%$ in adult contacts of infected children in day-care centres. ${ }^{2}$ Widespread previous exposure or infection has been documented by several sero-epidemiological surveys revealing high prevalence of specific anticryptosporidial IgG in all paediatric and adult populations studied thus far. ${ }^{11,12}$

Attempts to control environmental sources of infection have been thwarted by the resilient oocysts which can withstand extremes of temperature as well as standard disinfectants and chlorine. Particularly worrisome is the inability of water purification systems to eliminate Cryptosporidium. ${ }^{13,14}$ The oocyst is only $4 \mu \mathrm{m}$ in diameter, making it difficult to clear through filtration. ${ }^{1}$ Communitywide outbreaks, such as the one in Milwaukee, US, which affected more than 400000 people, have been traced to the public water supply. ${ }^{15}$

\section{Clinical manifestations}

Acute infection is characterised by watery diarrhoea with 6-12 bowel movements/day, crampy epigastric pain, weight loss, anorexia, malaise and flatulence. Such symptoms are typical of diseases of the small bowel, the primary site of cryptosporidial infection. Nausea, vomiting and fever may also be present. A small subset of patients may present with colitis but primary 


\begin{tabular}{|l|}
\hline Epidemiology \\
\hline - ubiquitous parasite found worldwide \\
- common cause of diarrhoea \\
- prevalence in AIDS: $5 \%$ (US and \\
Europe), $30-50 \%$ (tropical regions) \\
- infection $5-22$ days after ingestion \\
of as few as 30 oocysts \\
- sources of oocysts include sick \\
contacts, animals, soil, \\
contaminated food and water \\
\hline
\end{tabular}

Box 2

\section{Diagnosis \\ - demonstration of oocysts in fecal or intestinal biopsy specimens by special stains or immunological assays \\ - commonly used stains include acid fast (for fecal matter) and haematoxylin and eosin (for intestinal biopsy material) \\ - if clinical suspicion is high and initial stool exam is negative, repeat stool studies given variation in oocyst shedding in serial stool specimens}

Box 3

\begin{tabular}{|l|}
\hline Clinical manifestations \\
\hline - in normal hosts and HIV-infected \\
persons with CD $>200$, infection \\
usually leads to diarrhoeal syndrome \\
with spontaneous resolution \\
- in HIV-infected persons with \\
CD4 $<100$, infection most \\
commonly becomes chronic and \\
leads to an enteritis and, less \\
commonly, cholangitis \\
- voluminous, non-bloody diarrhoea \\
(6->20 bowel movements/day) \\
other signs/symptoms may include: \\
nausea, vomiting, crampy \\
abdominal pain, right upper \\
quadrant pain, fever, jaundice, \\
wasting/weight loss \\
\hline
\end{tabular}

Box 4

\begin{tabular}{|l|}
\hline Treatment \\
\hline - oral or intravenous fluid repletion or \\
maintenance \\
- nutritional supplements: oral, \\
enteral, parenteral \\
- antimotility agents \\
In persistent infection: \\
- optimize antiretroviral therapy \\
improvement in $50 \%$ of patients but \\
rarely eradicates parasite)
\end{tabular}

involvement of the large bowel is rare. Though the severity of symptoms may vary from individual to individual, a consistent finding is the spontaneous resolution of diarrhoea in the immunocompetent and well-nourished individual, usually within two weeks.

Two clinical patterns exist for HIV-related cryptosporidiosis: those persons with relatively preserved immunological function are able to clear infection, whereas those with depressed CD4 counts (ie, $\leqslant 180$ cells $/ \mathrm{mm}^{3}$ ) are usually chronically infected. ${ }^{16}$ Among persons with CD 4 counts $<100$ cells $/ \mathrm{mm}^{3}$, a few individuals $(<10 \%)$ may have spontaneous resolution but the remainder have life-long diarrhoea of a waxing and waning nature, malabsorption and wasting. The chronicity and severity of the diarrhoea are usually what prompt HIVinfected individuals to seek medical treatment. In two clinical studies involving patients in the US and Mexico, clinical presentations were remarkably similar, with duration of symptoms averaging 9-12 weeks and weight loss at presentation averaging $14 \mathrm{~kg} .{ }^{17,18}$

A particularly worrisome complication seen in over $10-15 \%$ of $\mathrm{HIV}$-infected persons with cryptosporidiosis is infestation of the biliary tree leading to sclerosing or acalculous cholangitis. ${ }^{2}$ Clinical manifestations may include fever, right upper quadrant pain, jaundice, nausea and vomiting, with or without diarrhoea. Diagnostic work-up should include liver enzymes (alkaline phosphatase may be elevated up to 25 times the upper limit), noninvasive (ultrasound) and occasionally invasive (cholangiography) imaging. ${ }^{19,20}$ Persistence of the organism in the biliary tract may lead to seeding of the intestine and prolongation of diarrhoea. Though rarer, other clinical manifestations include pancreatitis, probably due to infection of the pancreatic duct and hepatitis.

\section{Diagnosis}

Cryptosporidiosis is diagnosed by identifying oocysts in stool or gastrointestinal biopsy specimens. Though many stool identification techniques exist, the most widely used method is the acid-fast stain (cold Kinyoun modified, hot Kinyoun, Ziehl-Neelsen). ${ }^{1}$ Oocysts stain red with varying intensity allowing differentiation from similarly sized and shaped yeast which are not acid fast. Other studies, such as the direct immunofluorescent assay which utilises a monoclonal antibody, may increase the sensitivity of stool identification. If initial studies are negative, the optimum number of stool specimens for diagnosis is unknown. Serial stool specimens in HIV-infected persons reveal 10-fold variation in cryptosporidial oocyst shedding. ${ }^{21}$ The importance of obtaining multiple specimens (eg, at least three specimens on three separate days) is supported by studies on other protozoa that demonstrate increased yield with additional stool samples. ${ }^{22}$

If stool studies are negative, endoscopy should be considered to establish a definitive diagnosis when diarrhoea is refractory to antimotility agents or when the disease process changes (eg, development of colitic symptoms, bloody diarrhoea, right upper quadrant pain). Endoscopic biopsy specimens may reveal Cryptosporidium when stool studies have been negative in $<10 \%$ of patients. Other pathogens such as cytomegalovirus or Microsporidia may also be identified. ${ }^{18}$ When examining intestinal biopsy material, Cryptosporidia can be identified on the brush border of the mucosal epithelial surface and appear basophilic with haematoxylin and eosin staining. Failure to identify Cryptospor$i d i a$ in intestinal biopsy material does not rule out diagnosis, as cryptosporidial distribution may be patchy. ${ }^{23}$ Other identification methods include concentration techniques such as Sheather's sugar flotation.

\section{Treatment}

\section{NUTRITIONAL AND FLUID SUPPORT}

Cryptosporidial diarrhoea is voluminous and individuals may lose up to $17 \mathrm{1} /$ day in stool. ${ }^{24}$ Fluid loss is especially prominent in debilitated individuals who are unable to drink. Utmost attention needs to be paid to correction of electrolyte abnormalities and prevent dehydration with intravenous or oral rehydration solutions (ORS). A simple home-made ORS is $200 \mathrm{ml}$ of water with an eighth of a teaspoon of salt and two teaspoons of sugar. Individuals should be encouraged to start ORS early to prevent even initial dehydration.

Optimum nutritional support should include a trial of oral nutritional supplements before considering total parenteral or intravenous hyperalimentation. Nutritional supplements containing medium chain fatty acids may be better absorbed in patients with small intestinal injury and malabsorption. Milk and dairy products should be avoided since lactose intolerance is common. 


\begin{tabular}{|l|}
\hline $\begin{array}{l}\text { Possible strategies to } \\
\text { decrease risk of } \\
\text { Cryptosporidium transmis- } \\
\text { sion }\end{array}$ \\
\hline - use boiled or bottled water \\
- filter water utilizing $1-\mu \mathrm{m}$ filters \\
- employ enteric precautions when in \\
contact with persons with presumed \\
or proven cryptosporidiosis
\end{tabular}

Box 6
SPECIFIC ANTICRYPTOSPORIDIAL DRUGS

Though more than 100 drugs have been tried, none has proven uniformly effective in treating cryptosporidiosis. ${ }^{25}$ Paromomycin, a nonabsorbable aminoglycoside, has been shown to improve symptoms in approximately $50 \%$ of patients but complete resolution of diarrhoea with eradication of parasite occurs in only $10 \%$ of individuals. ${ }^{17}$ Other agents that have been tried with unencouraging results include the macrolide antibiotics spiramycin and azithromycin. ${ }^{25}$

Another therapeutic approach has been the use of immune-based therapies such as hyperimmune bovine colostrum and bovine transfer factor. These agents are produced by inoculating cows with cryptosporidial oocysts and isolating specific anticryptosporidial antibody or transfer factor from the cows' milk or lymph nodes, respectively. Trials have been limited by small numbers of patients, cumbersome production techniques and difficulty in standardising different batches of product. ${ }^{26,27}$

\section{ANTIRETROVIRAL AGENTS}

Although resolution of cryptosporidial infection with zidovudine has been documented in case report format, no studies have shown a definitive benefit of therapy in cryptosporidiosis. ${ }^{28}$ At present, combination antiretroviral therapy including protease inhibitors is recommended by the authors in the hope that the ensuing boost in immune function will help combat cryptosporidial infection.

\section{ANTIMOTILITY AGENTS}

Antimotility agents should be used for symptomatic relief and include first-line agents such as loperamide and diphenoxylate/atropine (lomotil). A stronger agent, tincture of opium, can be used for refractory diarrhoea with the warning that over-use may lead to hypomotility, constipation and nausea. Antimotility agents should be stopped immediately if bloody diarrhoea or fever develops and should not be restarted until the presence of invasive organisms has been ruled out. Trials of other antimotility agents such as octreotide, a somatostatin analogue, have not shown them to be more effective than standard agents in controlling diarrhoeal symptoms. ${ }^{29}$

\section{Conclusions}

Cryptosporidium is one of multiple emerging pathogens that poses considerable challenges to medical science. As populations of immunocompromised persons grow, it will cause increasing morbidity due to chronic severe enteritis. Innovative methods to treat this infection are urgently needed. Immunotherapeutic trials with orally administered Cryptosporidium polyclonal or monoclonal antibodies are ongoing. Novel pharmacologic approaches based on a better understanding of the biology of this protozoa may be effective. A successful vaccine to induce mucosal immunity would be a great advance.
1 Flanigan TP, Soave R. Cryptosporidiosis. In: Sun T, ed. Progress in clinical parasitology. New York: Springer-Verlag, 1993; pp 1-20.

2 Ungar BLP. Cryptosporidium and cryptosporidiosis. In: Broder S, Merigan TC, Bolognesi D, idiosis. In: Broder S, Merigan TC, Bolognesi D,
eds. Textbook of AIDS medicine. Baltimore, MD: Williams and Wilkins, 1994; pp 617-28.

3 McGowan I, Hawkins AS, Weller IVD. The natural history of cryptosporidial diarrhea in HIV-infected patients. AIDS 1993; 7: 349-54.

4 Dupont HL, Chappell CL, Sterling CR, et al. The infectivity of Cryptosporidium parvum in healthy volunteers. $N$ Engl $f$ Med 1995; 332: $855-9$.

5 Forgacs $\mathrm{P}$, Tarshis $\mathrm{A}, \mathrm{Ma} \mathrm{P}$, et al. Intestinal and bronchial cryptosporidiosis in an immunodeficient homosexual man. Ann Intern Med 1983; 99: $793-4$

6 Genta RM, Chappell CL, White AC, Kimbal KT, Goodgame RW. Duodenal morphology and intensity of infection in AIDS associated cryptosporidiosis. F Infect Dis 1993; 105: 1769-75.

7 Flanigan TP. Human Immunodeficiency Virus infection and cryptosporidiosis: protective immune responses. Am $\mathcal{F}$ Trop Med Hyg 1994; 50: 29-35

8 Heyworth MF. Immunology of Giardia and Cryptosporidium infections. F Infect Dis 1992; 166: $465-72$.
9 Jacyna MR, Parkin J, Goldin R, Baron JH. Protracted enteric cryptosporidial infection in selective immunoglobulin A and saccharomyces selective immunoglobulin A and saccharom

10 Lasser KH, Lewin KJ, Ryning FW. Cryptosporidial enteritis in a patient with congenital idial enteritis in a patient with congenital 10: $234-40$

11 Ungar BLP, Mulligan M, Nutman TB. Serologic evidence of Cryptosporidium infection in US volunteers before and after Peace Corps service in Africa. Arch Intern Med 1989; 149: 849-57.

12 Ungar BLP, Gillman RH, Lanata CF, et al. Seroepidemiology of Cryptosporidium infection in two Latin American populations. F Infect Dis 1988; 157: $551-5$.

13 McAnulty J, Fleming DW, Gonzalez AH. A community-wide outbreak of cryptosporidiosis community-wide outbreak of cryptosporidiosis 1994; 272: 1597-600.

14 Hayes EB, Matte TD, O'Brien TR, et al. Large Hayes EB, Matte TD, O'Brien TR, et al. Large
community outbreak of cryptosporidiosis due to community outbreak of cryptosporidiosis due to contamination of filtered public
Engl f Med 1989; 320: $1372-6$.

15 MacKenzie WR, Hoxie NJ, Proctor ME, et al. A massive outbreak in Milwaukee of Cryptosporidium infection transmitted through the public water supply. $N$ Engl $\mathcal{H}$ Med 1994; 331: 161-7.
16 Flanigan TP, Whalen C, Turner J, et al. Cryptosporidium infection and CD4 count. Ann Intern Med 1992; 116: 840-2.

17 Flanigan TP, Ramratnam B, Greaber C, et al. Prospective trial of paromomycin for cryptosporProspective trial of paromomycin for cryptospor-

18 Correa AL, Ramratnam B, Calva J, et al. Gastrointestinal cryptosporidiosis in HIV infected Mexican patients: analysis of 72 cases. $X I$ Intermational Conference on AIDS. Vancouver, Canada, July 1996. Abstract Pub B 1056.

19 Cello JP. Acquired immunodeficiency syndrome cholangiopathy: spectrum of disease. Am $\mathcal{F}$ Med 1989; 86: 539-46.

20 Dolmatch BL, Laing FC, Federle MP, Jeffrey RB, Cello JP. AIDS-related cholangitis: radiographic findings in nine patients. Radiology 1987; 163: $313-6$

21 Goodgame RW, Genta RM, White AC, Chappell CL. Intensity of infection in AIDS related pell CL. Intensity of infection in AIDS related

22 Hiatt RA, Markell EK, Ng E. How many stool examinations are necessary to detect pathogenic intestinal protozoa? Am f Trop Med Hyg 1995; 53: $36-9$.

23 Genta RM, Chappell CL, White AC, Kimball KT, Goodgame RW. Duodenal morphology and intensity of infection in AIDS-related cryptosporidiosis. Gastroenterology 1993; 105: 1769-75. 
24 Peterson C. Cryptosporidiosis in the patient infected with the Human Immunodeficiency Virus. Clin Infect Dis 1992; 15: 903-9.

25 Ritchie DJ, Becker ES. Update on the management of intestinal cryptosporidiosis in AIDS. Ann Pharmacother 1994; 28: 767-78.

26 Ungar BLP, Ward DJ, Fayer R, Quinn CA. Cessation of Cryptosporidium associated diarrhea in an acquired immunodeficient syndrome patient after treatment with hyperimmune bovine colostrum. Gastroenterology 1990; 98: 485-9.
27 McMeeking A, Borkowsky W, Klesius PH, Bonk $S$, Holzman RS, Lawrence HS. A controlled trial of bovine dialyzable leukocyte extract for cryptosporidiosis in patients with AIDS. 7 Infect Dis 1990; 161: $108-12$

28 Chandrasekar PH. 'Cure' of chronic cryptosporidiosis during treatment with AZT in a patient with the Acquired Immunodeficiency Syndrome. Am f Med 1987; 83: 187.
29 Simon DM, Cello JP, Valenzuela J, et al. Multicenter trial of octreotide in patients with refractory acquired immunodeficiency syndrome-associated diarrhea. Gastroenterology 1995; 108: $1753-60$.

\section{Medical Anniversary \\ JOHN COAKLEY LETTSOM, 22 November 1744}

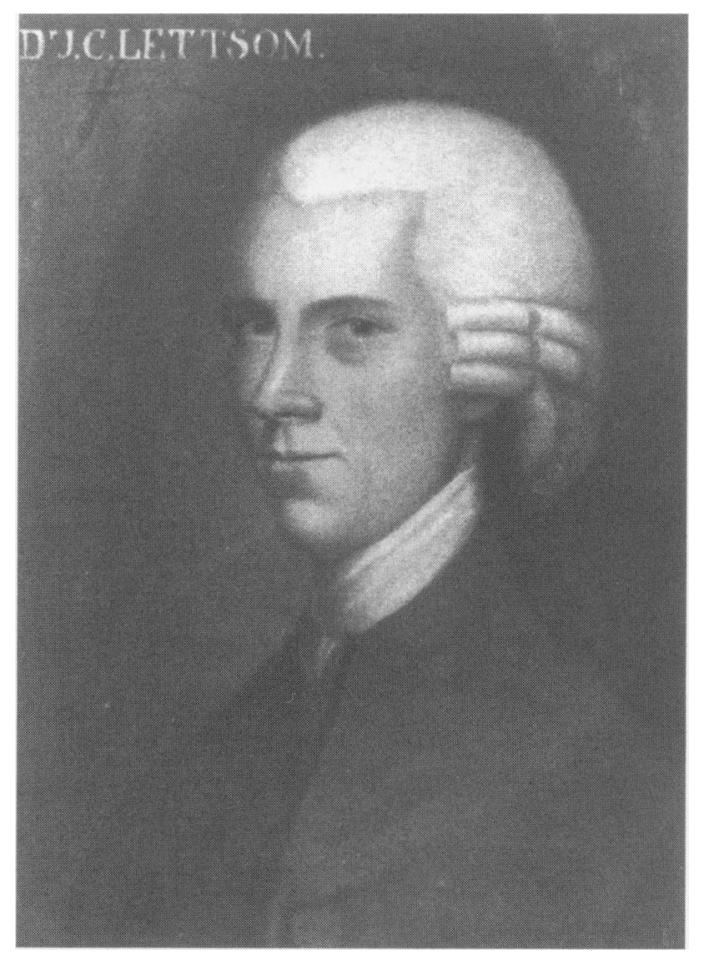

Photo courtesy of The Medical Society of London

John Coakley Lettsom (1744-1815) was born in Tortola, British Virgin Islands, less than $160 \mathrm{~km}$ east of Puerto Rico. He was one of the last of seven pairs of twins, and he and his brother Edward were the only pair to survive infancy. Their father was a plantation owner so he was able to send his son to a Quaker school in Lancashire. A year as a dresser at St Thomas' Hospital was eventually followed by medical education at Edinburgh and Leiden and a London practice with Dr John Fothergill, London's most celebrated Quaker physician. He and his wife Ann Miers had eight children and lived in his country residence The Grove, Camberwell. He became FRCP (Ed) in 1771 and FRS in 1773. His immortality is linked with the Medical Society of London, which he founded in 1773 . He also founded the Sea-bathing Infirmary, Margate. After a full and satisfying life helping others, Lettsom died on 1 November 1815 and is buried at the Quaker Burial Ground, Bunhill Row, London.

He was blessed with a sense of humour as revealed in this epigram against himself:

When any sick to me apply

I physics, bleeds and sweats 'em

If after that they choose to die,

Why verily! 